\title{
Functional lumen imaging of the gastrointestinal tract
}

\author{
Christian Lottrup ${ }^{1} \cdot$ Hans Gregersen ${ }^{2}$ Donghua Liao ${ }^{3}$ Lotte Fynne ${ }^{4}$. \\ Jens Brøndum Frøkjær ${ }^{5,6} \cdot$ Klaus Krogh $^{4} \cdot$ Julie Regan $^{7} \cdot$ Peter Kunwald $^{1}$. \\ Barry P. McMahon ${ }^{2,7}$
}

Received: 16 March 2015 / Accepted: 28 April 2015/Published online: 16 May 2015

(C) Springer Japan 2015

\begin{abstract}
This nonsystematic review aims to describe recent developments in the use of functional lumen imaging in the gastrointestinal tract stimulated by the introduction of the functional lumen imaging probe. When ingested food in liquid and solid form is transported along the gastrointestinal tract, sphincters provide an important role in the flow and control of these contents. Inadequate function of sphincters is the basis of many gastrointestinal diseases. Despite this, traditional methods of sphincter diagnosis and measurement such as fluoroscopy, manometry, and the barostat are limited in what they can tell us. It has long been thought that measurement of sphincter function
\end{abstract}

Hans Gregersen

hag@giome.org

Christian Lottrup

chlo@rn.dk

1 Mech-Sense, Department of Gastroenterology and Hepatology, Aalborg University Hospital, Aalborg, Denmark

2 GIOME and the Key Laboratory for Biorheological Science and Technology of Ministry of Education, Bioengineering College of Chongqing University, 83 Shabei Lu, 400044 Chongqing, China

3 GIOME Academia, Department of Medicine, Aarhus University, Aarhus, Denmark

4 Neurogastroenterology Unit, Department of Hepatology and Gastroenterology, Aarhus University Hospital, Aarhus, Denmark

5 Mech-Sense, Department of Radiology, Aalborg University Hospital, Aalborg, Denmark

6 Department of Clinical Medicine, Aalborg University, Aalborg, Denmark

7 Trinity Academic Gastroenterology Group, Tallaght Hospital, Dublin, Ireland through resistance to distension is a better approach, now more commonly known as distensibility testing. The functional lumen imaging probe is the first medical measurement device that purports in a practical way to provide geometric profiling and measurement of distensibility in sphincters. With use of impedance planimetry, an axial series of cross-sectional areas and pressure in a cathetermounted allantoid bag are used for the calculation of distensibility parameters. The technique has been trialed in many valvular areas of the gastrointestinal tract, including the upper esophageal sphincter, the esophagogastric junction, and the anorectal region. It has shown potential in the biomechanical assessment of sphincter function and characterization of swallowing disorders, gastroesophageal reflux disease, eosinophilic esophagitis, achalasia, and fecal incontinence. From this early work, the functional lumen imaging technique has the potential to contribute to a better and more physiological understanding of narrowing regions in the gastrointestinal tract in general and sphincters in particular.

Keywords Functional lumen imaging probe . Gastrointestinal sphincters · Geometric profiling

$\begin{array}{ll}\text { Abbreviations } \\ \text { CSA } & \text { Cross-sectional area } \\ \text { EGJ } & \text { Esophagogastric junction } \\ \text { EoE } & \text { Eosinophilic esophagitis } \\ \text { FLIP } & \text { Functional lumen imaging probe } \\ \text { GERD } & \text { Gastroesophageal reflux disease } \\ \text { GI } & \text { Gastrointestinal } \\ \text { LES } & \text { Lower esophageal sphincter } \\ \text { POEM } & \text { Peroral endoscopic myotomy } \\ \text { TIF } & \text { Transoral incisionless fundoplication } \\ \text { UES } & \text { Upper esophageal sphincter }\end{array}$




\section{Background and aims}

Gastrointestinal (GI) sphincters are specialized zones that serve to divide the GI tract into distinct segments, i.e., compartments. Sphincters appear as narrow regions of the lumen of the tract, most of the time being closed. The various segments of the GI tract, through which food and other luminal contents pass, are hollow low-pressure organs that are separated by specialized sphincteric circular muscles. These sphincters are used for temporary mechanical separation of compartments for controlling the direction and flow of semisolid and liquid contents. The physiological mechanism at the anatomical site plays a fundamental role in sphincter operation, such as how sphincter muscles contract and relax. Through neural circuits, changes in sphincter pressure are coordinated with smooth muscle contraction and relaxation in the organs on either side [1]. Simply put, sphincters or valvular regions in the GI tract provide a very important role in the proper control and transit of ingested food.

This article aims to review the literature on a new technology named the "functional lumen imaging probe" (FLIP) that was developed to test the competence of GI sphincters using bag distension in the organ of interest. The review sets out to provide an update on the work done with the FLIP in GI organs to date, in particular on sphincters. Focus is on the esophageal sphincters and the anal sphincter, on which most of the work on the FLIP has been published.

\section{Methods}

For this nonsystematic review, we conducted a search (November 2014) of PubMed and EMBASE with the following search words: "functional lumen imaging probe," "FLIP," "EndoFLIP," "impedance planimetry," and "distensibility." Mainly, full-length articles were considered, but some conference abstracts and book chapters were included as well. To the best of the authors' knowledge, the research on which this review is based, including that of the authors themselves, was conducted in accordance with common ethical standards and the Declaration of Helsinki.

\section{Technical improvements and development in assessment of GI sphincters}

Traditionally, sphincters were studied by pull-through and stationary manometry techniques, by the Dent sleeve, and more recently, using high-resolution manometry or high- definition manometry [2-4]. Manometry and the Dent sleeve proved useful for showing phenomena such as transient lower sphincter relaxations and increased lower esophageal sphincter (LES) tone in patients with achalasia, and have gained widespread use in physiology and clinical laboratories around the world. However, data of this kind have shown a poor correlation with the strength and competence of sphincters [5, 6]. For measurement in the GI tract, distensibility is usually defined as the relationship between the minimum cross-sectional area (CSA) in the narrow region and the bag (noncompliant balloon) pressure at the same point. The FLIP method is a good practical method to measure distensibility in the sphincters and luminal regions of the GI tract, since it easily captures the geometry of the whole sphincter region because of multiple concomitant measurements.

The underlying principle for the FLIP method, impedance planimetry, was presented by Gregersen et al. [7] in 1988 for combined pressure and CSA measurements. Simply put, "impedance planimetry" refers to using electrical impedance to measure area. In the case of lumens in the GI tract, this is the CSA in the distending bag at the position of the measurement electrodes. In early studies, impedance planimetry was not considered suitable for studying sphincteric regions owing to the potential for dislocation of the then short bag and the single CSA measurement [8]. Nevertheless, some successful measurements by means of impedance planimetry were made in the LES and the anal sphincter [8,9]. The barostat, a technology based on pressure-volume measurements, is a competing distension technology that has mainly been used in the stomach and tubular segments of the GI tract. However, it is not considered useful for studying sphincters, since the geometric volume distribution in the bag is not known. Although use of it will provide a measurement of pressure and volume, it cannot be precisely aligned with an objective measure of sphincter size and function, nor can it be relied on to provide an accurate geometric image of function in this region [10]. Other groups led by Shaker, Mittal, and Pandolfino [1113] independently developed other distension devices, but use of none of these has become common practice because of limitations caused by use of radiation, the degree of technical difficulty, and geometry-related factors. A major step forward in the development of impedance planimetry was made by Gregersen and coworkers [14] from 2004 and onward, where they developed several new designs of probes capable of making multiple geometric measurements and with improved electrode designs and signal conditioning for rectal studies and early FLIP studies by McMahon et al. [15-17] on the esophagogastric junction (EGJ). 


\section{Technical basis of the FLIP method}

The basic principle of the FLIP method is that of impedance planimetry [18], i.e., measurements of the CSA inside a distending bag from electrical impedance measurements and of intrabag pressure. The FLIP has several electrodes, and is therefore able to measure a longitudinal series of equally spaced CSAs inside the bag. Currently, the standard is 16 CSA measurements. Figure 1 shows an example of equipment used in the FLIP method.

For the first time a straightforward technique capable of gathering real-time, objective data on the actions of sphincteric regions while provoked by distension was made available to GI tract clinicians. An innovative representation method was made possible as semi-3D geometric plots. This can be viewed in Fig. 2, where CSAs at fixed intervals along the catheter inside the fluid-filled bag are represented along an axis as estimated diameters. This is the classic FLIP representation. Figure 3 shows a different and newer representation of FLIP data with a schematic of a 16-CSA-measurement probe with two excitation electrodes and 16 sequential pairs of detection electrodes (left panel). The left panel in Fig. 3 also shows representative tracings of 16 CSAs from distension of the anal canal, and the right panel shows the data represented as color contour plots as devised by Gregersen et al. [19].

Despite the fact that the FLIP was originally developed for studying the EGJ, it also found use in other sphincters and in nonsphincteric regions. It has also been tested as a diagnostic technology and a guiding technology during endoscopic or surgical procedures (see later).

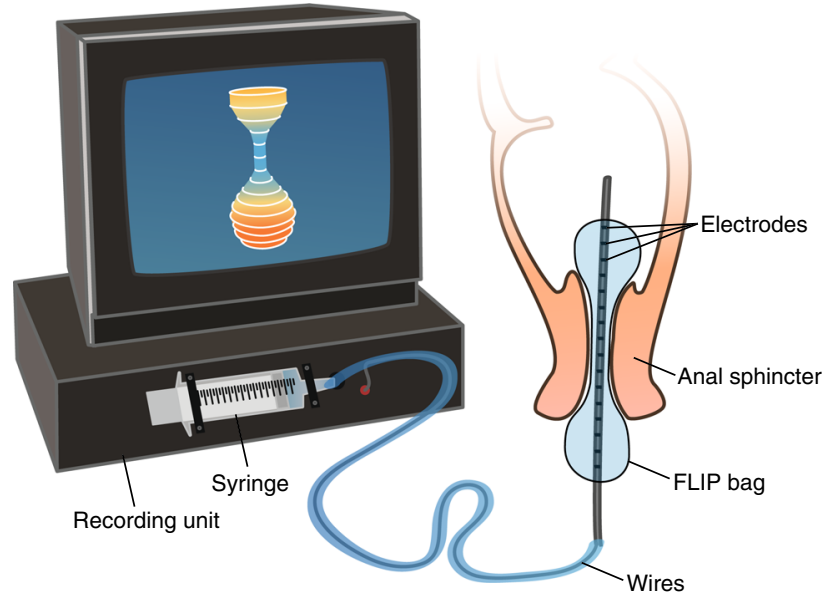

Fig. 1 An example of equipment used in the functional lumen imaging probe $(F L I P)$ method, with the probe placed in the anal sphincter and connected to the recording unit. The probe bag is filled with saline from the syringe through the connecting catheter. Live images of the data generated by the probe are visible on the screen

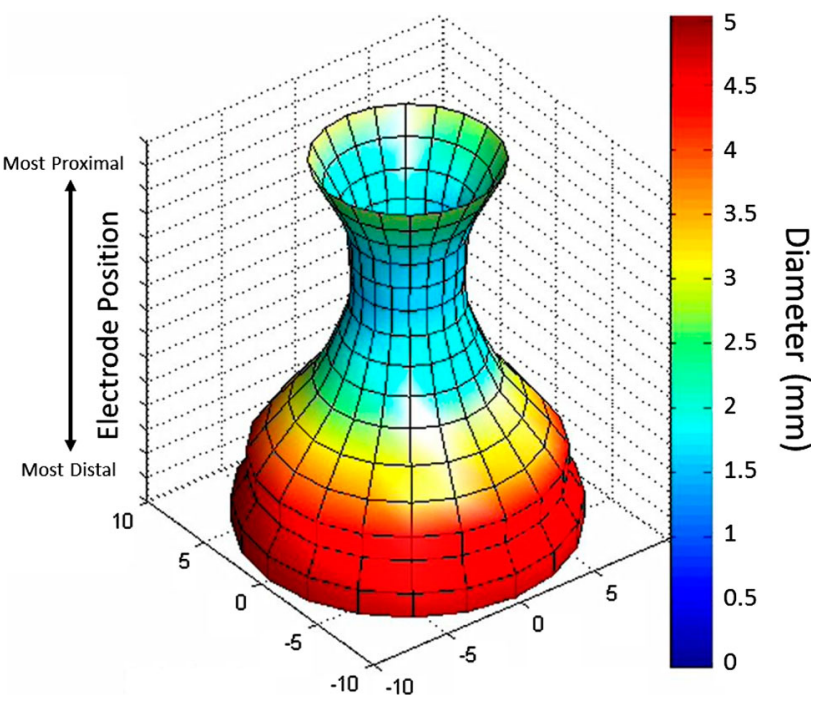

Fig. 2 Classic functional lumen imaging probe image from files of McMahon and Gregersen showing how cross-sectional area data from the esophagogastric junction can be simulated as a three-dimensional profile of estimated diameters indicated by color changes from blue (smaller diameters) through green, yellow, and then red (larger diameters). The oral direction is upward

\section{Upper esophageal sphincter}

Video fluoroscopy is the gold standard for measuring upper esophageal sphincter (UES) function, yet it is an extremely subjective test and the outcome depends to a large extent on the practitioner [20]. Even for experienced examiners, the use of video fluoroscopy and swallow studies to determine and quantify the extent of movement and opening in the pharyngoesophageal segment region of the UES can be very challenging. Patients with stroke sequelae and severe neurological disorders such as Parkinson's disease are still liable to have the effect of their condition on the proper functioning of the UES imprecisely diagnosed when video fluoroscopy is used [21]. The opening time of the UES is no more than 0.4-0.6 s. Apart from for a few trained and very experienced experts, examiners find this very difficult to observe in a live test, and the image must be played back usually at a slow speed to observe movement and opening patterns [22]. High-resolution manometry provides a better representation of tone or closure of the UES, and is often used along with endoscopy. It has been suggested that it can define function of the UES [23], but it cannot give information on characteristics and function of the region during opening as previously described for the EGJ [24].

Recent studies have demonstrated that the FLIP can be safely placed and distended in the region of the UES despite its proximity to the trachea. This gives the potential for a tool with the ability to profile UES opening and relaxation activity during bag distension and swallowing events. Studies have shown that the opening patterns that occur during 


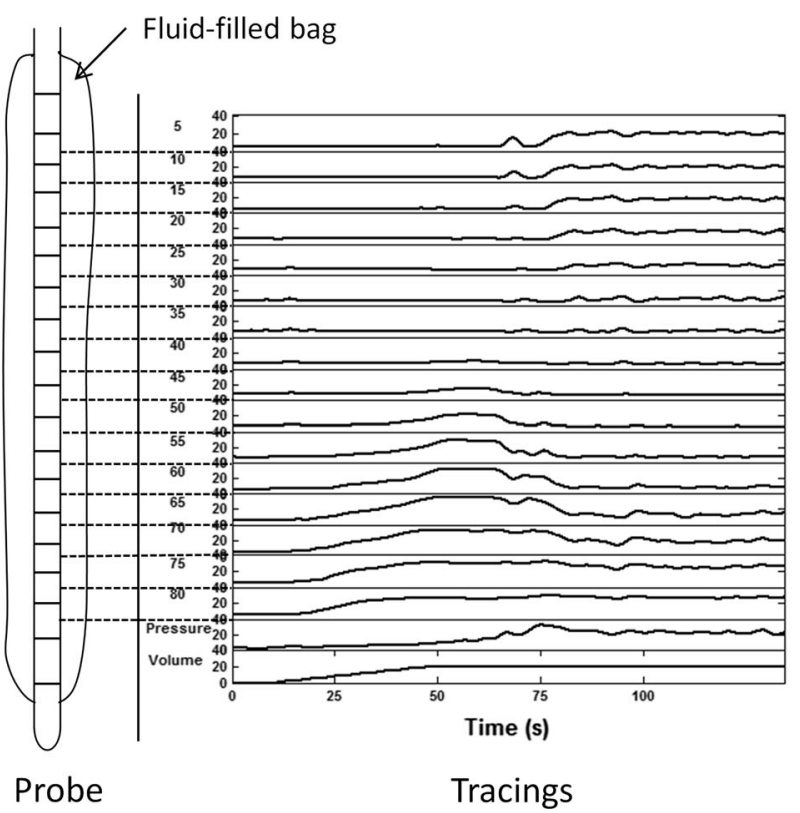

Fig. 3 On the left, a simple diagram of a fluid-filled functional lumen imaging probe showing how each of the 16 electrode pairs generates a tracing related to the dynamic changes in the cross-sectional areas as the bag fills, with time on the $x$-axis. Pressure and bag volume are also shown as tracings. The color contour plot on the right shows time on

distension and swallowing can be recorded accurately and objectively in healthy controls [25]. This is demonstrated well in Fig. 4, where on the left the FLIP probe can be clearly seen in position in the UES in fluoroscopic images from two healthy volunteers. On the right, the FLIP images show how the technique can be used to show opening during swallowing and lateral movement during a head turn. Furthermore, with the use of color contour plotting the timing, CSA changes can be visualized [26]. This has the potential to demonstrate in a new way the opening of the UES live as the studies are conducted and could complement the information provided by swallow studies [26]. The potential for using the FLIP to assist in total laryngectomy has also been explored. As expected, a reduction in UES tone in this group of patients was demonstrated after surgery [27].

Studies on the use of the FLIP in the UES are relatively new and require further investigation. This may unravel whether the technique could be effective in providing more information to aid with understanding, diagnosis, and treatment in the UES as it relates to the overall role of the UES in disease.

\section{Gastroesophageal reflux disease}

The anatomy and physiology of the EGJ have been studied extensively using endoscopy, barium esophagram, manometry, and magnetic resonance imaging [28-31]. In recent

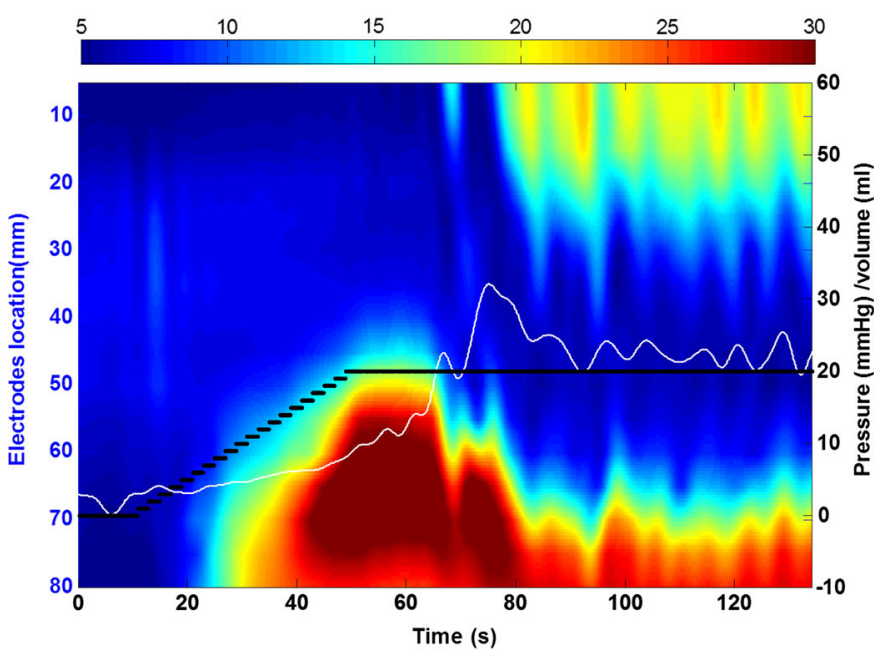

\section{Color contour plot}

the $x$-axis and electrode position on the left $y$-axis; color from the scale bar at the top shows smaller cross-sectional areas in blue, moving through green and yellow to red for larger areas. The white line represents bag pressure and the black line represents controlled bag volume, with the units on the right $y$-axis

years, several studies have also used the FLIP to characterize the functional properties of the esophagus and the EGJ. First, McMahon et al. [15-17] showed the feasibility of the FLIP for evaluation of the EGJ. A few years later, Beaumont et al. [32] examined Barrett's esophagus patients before and after radio-frequency ablation, and found the EGJ distensibility unchanged after the procedure. Later, Kwiatek et al. [33] found the CSA in the EGJ and the distensibility index to be increased twofold to threefold in gastroesophageal reflux disease (GERD) patients compared with controls. Kwiatek et al. [34] examined fundoplication patients between 4 months and 7 years postoperatively, and found decreased distensibility of the EGJ during the deglutitive period compared to controls. They indicated that the FLIP method will be a valuable method to assess the outcome of antireflux surgery as a supplement to other measured outcomes such as remaining reflux symptoms and side effects such as dysphagia and bloating. This was later investigated by Ilczyszyn and Botha [35], who performed FLIP measurements of the EGJ at six different time points before, during, and after fundoplication procedures in 17 reflux patients. The main findings were twofold to threefold increased distensibility in hiatus hernia patients at the baseline compared with patients without hiatus hernia and threefold to fourfold decrease in EGJ distensibility immediately after fundoplication. Correlation was not found between FLIP findings and clinical parameters such as reflux symptoms and acid exposure. This is in contrast to 
Fig. 4 Still images from fluoroscopic studies of the functional lumen imaging probe $(F L I P)$ in position in the upper esophageal sphincter $(U O S)$ of two healthy volunteer participants are shown on the left. The volunteer turns the head in the bottom image. The FLIP can clearly be identified by the electrode arrays. The right panel shows FLIP studies on these participants indicating the profiles at rest, with estimated minimum diameters identified, then the minimum diameter during dry swallows, clearly indicative of opening, and finally profiles for both with minimum diameters during right head turns, indicating a shift in either the probe or the narrow region
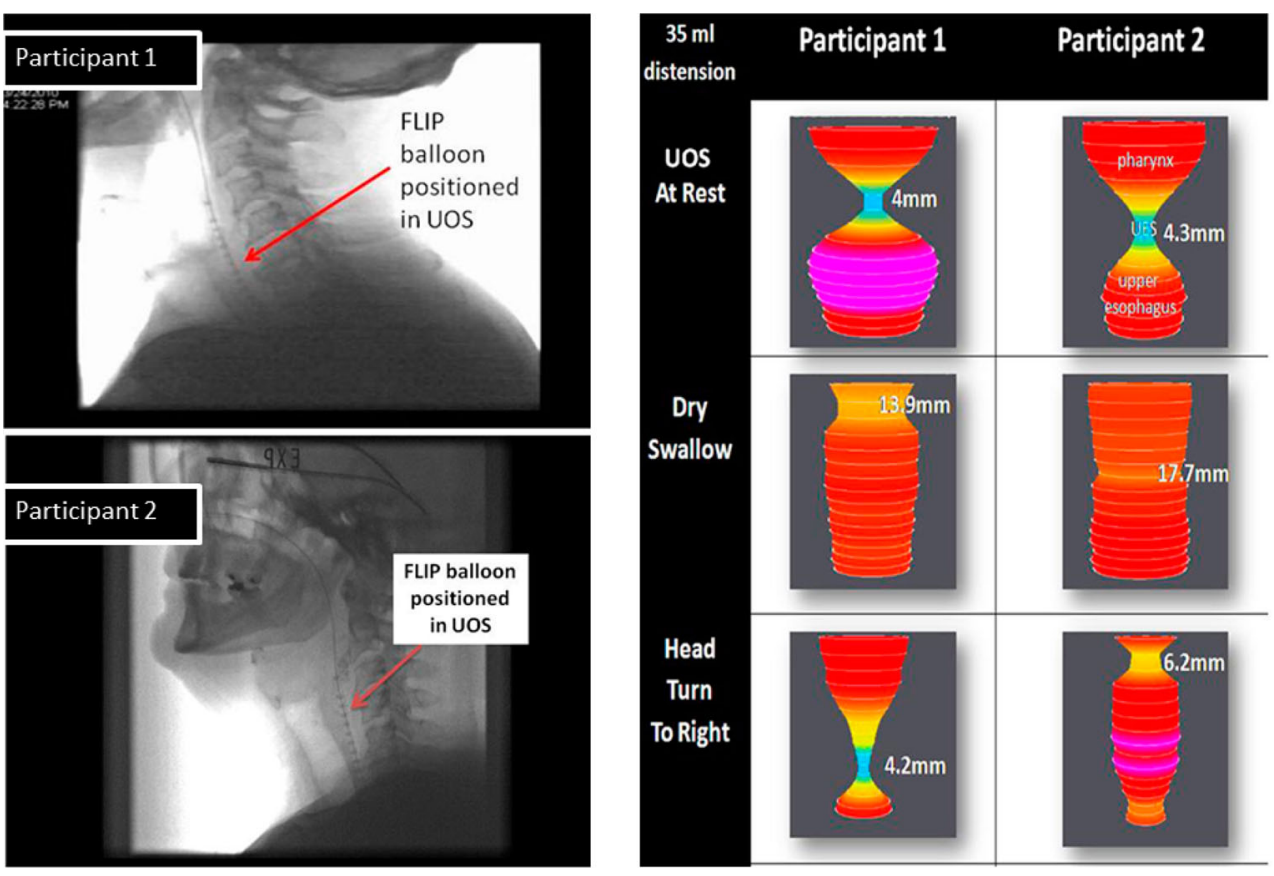

findings by Nathanson et al. [36], who studied 51 patients undergoing various laparoscopic surgical procedures with the FLIP before and after induction of pneumoperitoneum. They found a significantly increased distensibility after pneumoperitoneum. Hence, they emphasized the need to take this into account when using the FLIP, e.g., for targeted fundoplication procedures, as well as the need for further evidence. None of these studies involved long-term follow-up.

The FLIP has also been investigated for use in endoscopic antireflux surgery. The first use was in dogs, where controls were compared with two groups undergoing slightly different procedural techniques. Following the endoscopic antireflux surgery procedure known as transoral incisionless fundoplication (TIF), there was a significant decrease in CSA of the EGJ compared with the baseline and the CSA in the controls. However, the CSA of both groups returned to the baseline at the 2-week follow-up. The FLIP results correlated with $\mathrm{pH}$ testing results and measures of EGJ structural integrity [37]. In a later explorative clinical study primarily investigating the effect of TIF on clinical parameters in patients dissatisfied with medical treatment, Rinsma et al. [38] evaluated EGJ distensibility immediately before and after the TIF procedure. They concluded that TIF decreased EGJ distensibility and the reflux symptom score. However, clinical measures such as total acid exposure were not decreased postoperatively. No control group was included in either study, neither animal nor human.

Another study used the FLIP to characterize the functional properties of different esophageal regions. As expected, the least distensible locus was the EGJ, whereas the region 6-10 $\mathrm{cm}$ above the EGJ had higher distensibility, and the region $2-5 \mathrm{~cm}$ above the EGJ had the highest distensibility [39]. Looking into possible treatment options for GERD, Fukazawa et al. [40] found that mosapride given intravenously augmented peristaltic contractions, increased LES pressure, and decreased EGJ distensibility.

Recently, Lottrup et al. [41] investigated functional properties of the EGJ with the FLIP in a group of Barrett's esophagus patients with hiatus hernia and in controls. In line with earlier findings, increased distensibility in hiatus hernia patients was found. However, Lottrup et al. were also able to locate and measure the separate crural diaphragm and LES components of the hernia, and concluded that the crural diaphragm is the more distensible and thus less competent part. Furthermore, the FLIP was suggested as a possible tool for the diagnosis of hiatus hernia (Fig. 5).

Most of these publications report data demonstrating the value of the FLIP in measuring functional or biomechanical properties of the esophagus and the EGJ. However, the FLIP still needs to prove its definitive value in the clinical evaluation of patients with GERD and in patients being treated for GERD. In an attempt to address this issue, Tucker et al. [42] recently concluded that, when examining GERD patients and controls to which the investigators were blinded, the FLIP in the EGJ was not able to distinguish subjects with pathologic esophageal acid exposure from those with normal acid exposure. However, EGJ distensibility was increased in the GERD patient group compared with controls, and no analysis of FLIP findings 
Fig. 5 Functional lumen imaging probe images showing the visualization of different esophagogastric junction components. a The esophagogastric junction of a healthy volunteer. In a Barrett's esophagus patient with hiatus hernia, $\mathbf{b}$ the entire hiatus hernia region, $\mathbf{c}$ the lower esophageal sphincter $(L E S)$ component of the hiatus hernia, and $\mathbf{d}$ the crural diaphragm $(C D)$ component of a hiatus hernia are visualized. The oral direction is upward

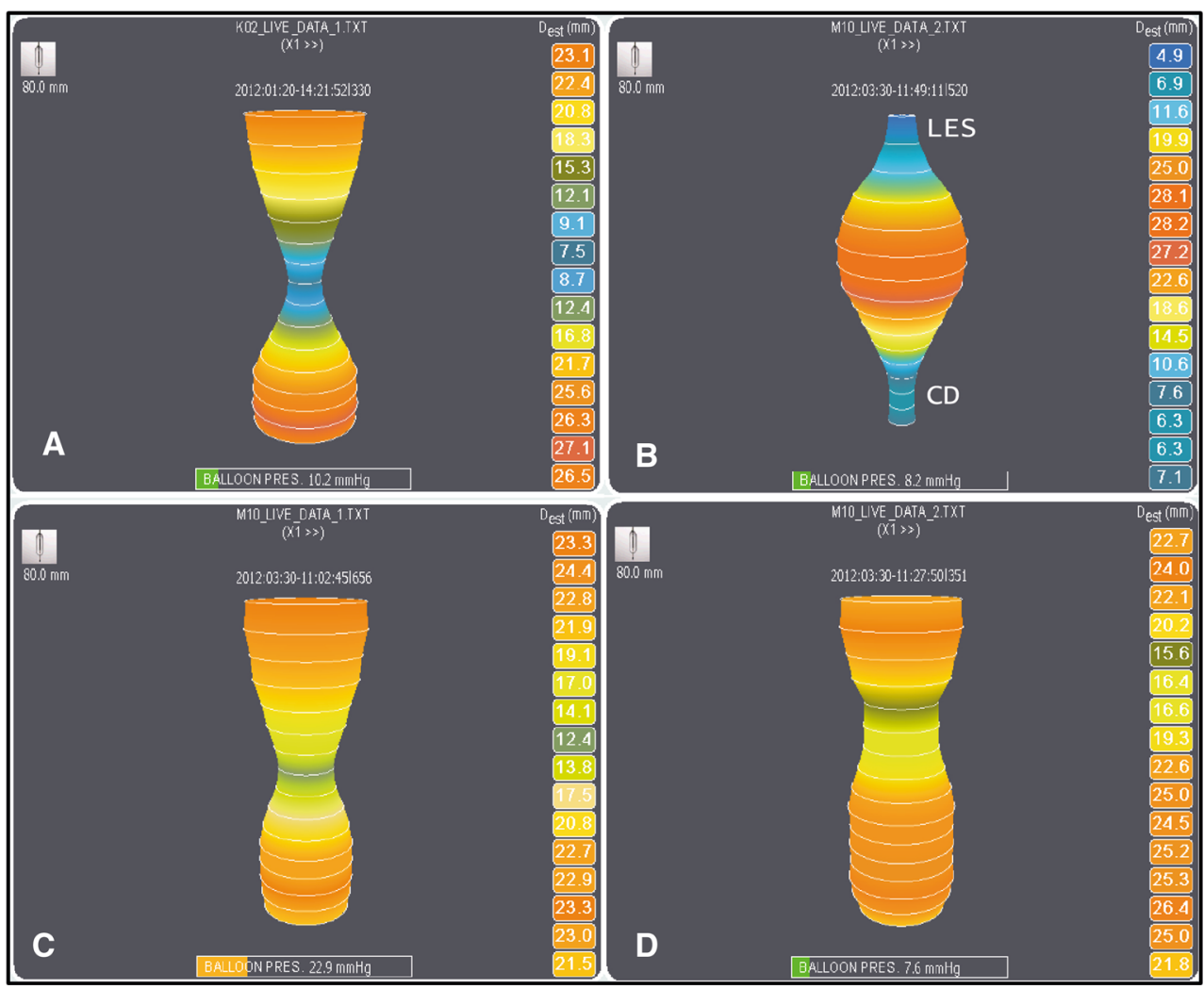

with respect to other clinical findings such as the symptom score was made. Other studies have found significant association with reflux symptoms $[38,41]$.

\section{Eosinophilic esophagitis}

Eosinophilic esophagitis (EoE) is an allergic inflammatory disease of the esophagus which was discovered quite recently. It is typically a chronic disorder that affects the structure and function of the esophageal lumen. The FLIP has shown some potential as a clinical tool for indirect measures in EoE. In 2011, Kwiatek et al. [43] investigated esophageal wall properties in EoE patients with the FLIP. Patients in whom EoE was confirmed by biopsy were found to have decreased distensibility of the esophageal body and EGJ compared with healthy controls. However, no direct association of FLIP parameters with established clinical parameters such as eosinophil count in biopsies, age, or sex was found. Traditional compliance measures using bag pressure versus estimated volume did not show significant differences between patients and healthy controls [43]. The same group later investigated the FLIP as a tool to predict the risk of food impaction in EoE [44]. They concluded that EoE patients had a lower maximal reachable CSA (socalled distension plateau) than controls and that this measure predicted the risk of food impaction. However, more studies are needed to determine if the FLIP is a useful tool in EoE diagnosis and monitoring.

\section{Achalasia}

Achalasia is a neuropathic disorder characterized by lack of relaxation of the EGJ with secondary aperistalsis and dilation oral to the EGJ. The FLIP has been used in several studies to provide new measures of EGJ properties in patients with achalasia. The studies concluded that the distensibility index of the LES is significantly lower in patients with achalasia than in healthy subjects [45-47] and symptoms seem to correlate better with distensibility assessed with the FLIP than with LES pressure assessed by manometry [46]. The standard surgical treatment of achalasia is laparoscopic Heller myotomy, whereas peroral endoscopic myotomy (POEM) is a recent and less invasive alternative. Studies agree that the distensibility index of the LES significantly increases after both Heller myotomy and POEM [47-50]. However, the increase in distensibility index appears to be larger after POEM than after Heller myotomy [49, 51].

In spite of surgery, symptoms often persist in a significant proportion of patients, whereas in others severe reflux returns. The first suggested intraoperative use of the FLIP for surgery was by Perretta et al. [52]. A more recent 
study has indicated that intraoperative measurement of the distensibility of the EGJ using the FLIP can predict the clinical outcome of Heller myotomy and POEM [51]. Thus, patients with the smallest increase in the distensibility index during surgery continued to have symptoms at follow-up, whereas those with the highest distensibility had increased risk of reflux symptoms. The study authors defined a range for the "ideal" distensibility index at the end of the surgical procedure. However, the findings were not confirmed in another study [50] and need to be replicated in future trials. Teitelbaum et al. [51] found that POEM could be restricted to the EGJ, whereas laparoscopic Heller myotomy needed to be extended further cephalad to normalize the distensibility index.

Very few centers have published their experiences with the FLIP in patients with achalasia, and the total number of patients included is relatively small. Their results, however, suggest that the FLIP is a useful tool for intraoperative assessment of the efficacy of POEM and Heller myotomy. Whether this will improve the outcome of surgery needs to be confirmed in future studies, before the application moves into widespread clinical use.

\section{Anal sphincters}

Anal continence, defecating, and passing gas are complex tasks depending on coordination between anal sphincter muscles, reflexes, anorectal motility and anorectal wall properties. Many diseases and conditions affecting these functions impede what is normal and lead to fecal incontinence or obstructed defecation. The underlying physiology of muscle function contributing to anorectal competence is complex. Most often, the muscle function of the anal canal is tested with anal manometry. However, maintaining continence and passing stool are dynamic properties and are not well represented with manometric measurements. Also, the structures surrounding the anal canal are not homogeneous, and therefore differ along the longitudinal axis. Furthermore, it has been proposed that resistance to distension, and not anal pressure, is the main determinant of anal continence [53].

The FLIP, spanning the whole length of the anal canal, provides detailed information on anal canal properties and the ability to resist distension in different segmental areas. So far, only four studies have been published [54-57]. In vitro tests have shown FLIP distensibility measurements to have great validity and reproducibility [54]. Dividing the anal canal into three segments in healthy volunteers demonstrated different distension properties in each segment during distension. First, the upper anal canal opened, which was followed by opening of the lower part and shortening of the middle, closed part. At higher volume loads, the middle part was also distended. This is consistent with the middle part of the anal canal being the least distensible (Fig. 6).

An additional study on volunteers showed a higher opening pressure for women than for men [55]. This suggests a different distensibility in men and women, but the findings were not consistent with earlier manometric findings. The FLIP study demonstrated consistent distension profiles in all 20 volunteers. Two studies evaluated the dynamic properties in the anal canal in patients with fecal incontinence [56, 57]. Comparing idiopathic fecal incontinence patients with healthy controls showed that the distensibility properties vary with the degree of distension [56]. The resistance to distension in the middle part was reduced in fecal incontinence patients, and the reduction was intensified with greater distension. In patients with systemic sclerosis, the middle part, representing the internal anal sphincter, was also the most resistant [57]. Compared with healthy controls, however, the distensibility in patients with systemic sclerosis was clearly increased (Fig. 6). This is consistent with knowledge that the internal anal sphincter is damaged in systemic sclerosis. This was confirmed by endoanal ultrasonography showing a thinning of the internal anal sphincter [58].

\section{Limitations and FLIP-measurement-based biomechanical assessment}

Like for all other measurement technologies, use of the FLIP is subject to limitations. Several studies have validated that the CSA and pressure measurements are accurate, and basically the equipment that has been used, noncommercial and commercial, has taken into account the possible sources of error when using impedance planimetry (reviewed by Gregersen [10]). However, it is strongly recommended for those who may develop their own system that they consult the early literature on impedance planimetry and the book by Gregersen [10].

It is worth noting that the FLIP measures CSAs rather than diameters. In other words, when the diameter is the outcome measure, it is based on the assumption that the lumen is circular; otherwise small errors may be induced. This has some implications for computation of more advanced mechanical parameters (see below). It is also worth noting that curvature of the lumen is not assessed, rather the FLIP considers it to be a straight structure. Another factor that should be considered is the fact that only one pressure measurement is done inside the bag. This poses no problem when the bag is being filled and consists of only one compartment, since the pressure will be the same everywhere inside the bag (except for minor hydrostatic effects). However, if the bag is divided into two 


\section{A Healthy subject}
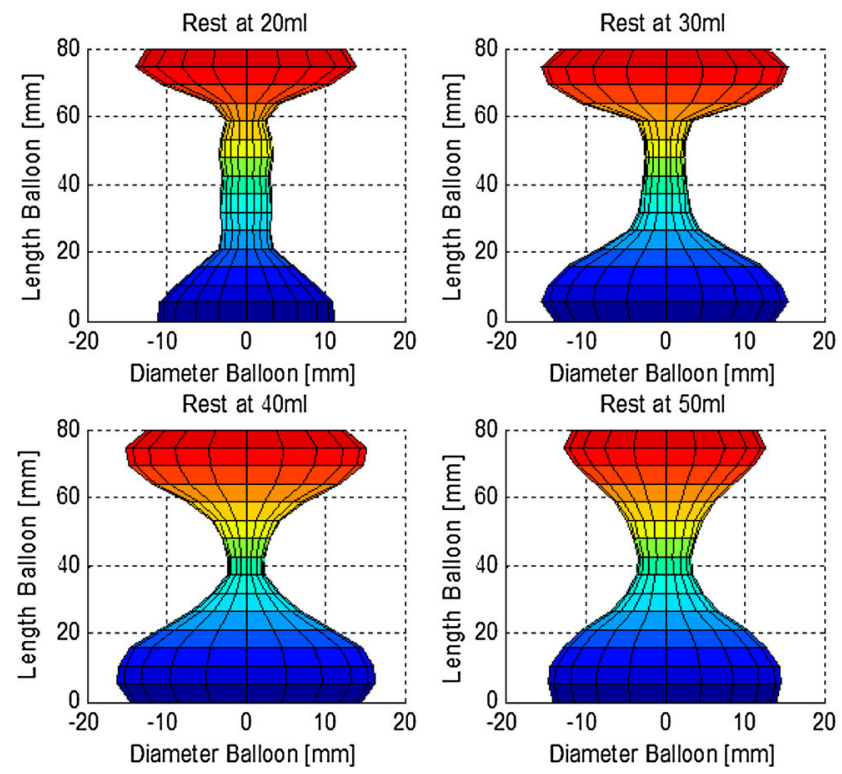

Fig. 6 Functional lumen imaging probe visualizations of the anorectal regions in $\mathbf{a}$ a healthy volunteer and $\mathbf{b}$ a patient with systemic sclerosis with the functional lumen imaging probe distended to bag

compartments-for example, by a narrow sphincter-then the pressure will be measured only in the compartment where the pressure sensor is located. It should be emphasized that the pressure can be higher or lower in the other compartment and likely will be much higher where the narrowing is. It would be an advantage if new systems were to implement at least two sensors.

Biomechanical assessment often deals with force-deformation properties of tissues. Manometry and endoluminal imaging by means of ultrasonography and magnetic resonance imaging are often used in detecting sphincter anatomy as well as sphincter (patho)physiology in health and in disease [59-64]. However, endoluminal imaging and pressure measurements in sphincters do not provide information on either geometry during distensions or mechanical properties as parameterized, for example, by length-tension relations, i.e., the force-deformation relationship produced by the sphincter muscles [65-67]. The length-tension relations are closely associated with the muscle function of the GI tract in different states and diseases. The FLIP provides the information needed to perform simplified mechanical analysis such as length-tension relationships from various locations along the sphincters. Figure 7 shows a simple diagram of pressure versus stretch ratio for three locations in the anal sphincter. Studies done with the FLIP demonstrate the nonlinear pressure (tension)-deformation relationships of GI sphincters [33, 54, $57,68]$. Often the pressure is observed to increase during
B Systemic Sclerosis patient
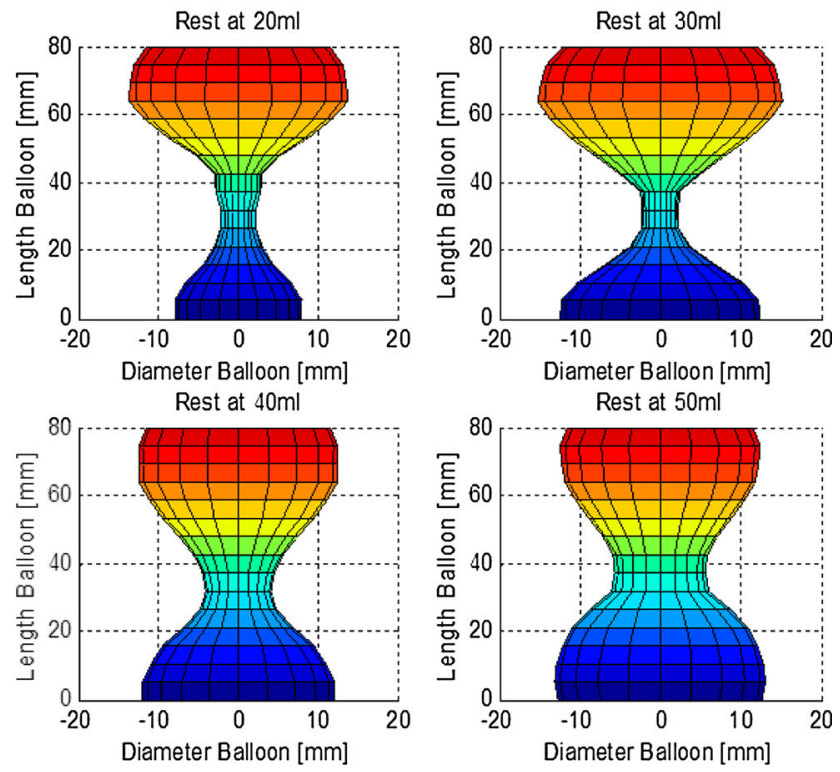

volumes of $20,30,40$, and $50 \mathrm{ml}$. Note the shorter canal length and the wider cross-sectional area at the same volumes in the systemic sclerosis patient

the beginning of the measurement without much distension of the sphincter, but at some point, which may be termed the "opening pressure," the sphincter starts to yield, after which it becomes much more compliant until a certain point. This is likely due to active relaxation, although it can be difficult to distinguish the active and passive contributions [10]. A good analogy for the passive contribution is blowing up a balloon. At first, it requires a high pressure, but when the balloon starts to distend, it actually becomes easier, because the tension increases, since it is a product of the pressure and the radius. At some point the balloon becomes stretched to a degree where the balloon material properties resist further distension.

In most of the studies published, the distensibility is evaluated in terms of very simple measures such as simply looking at the magnitude of the CSA or dividing the CSA by the pressure at a given point of distension [33, 54, 57]. This may easily introduce errors. Distensibility measures must take into account factors such as the initial conditions (pressure and CSA), the level of distension, and the actual slope of the mechanical data curves. In some studies the sphincter elasticity has been evaluated in terms of the pressure-strain modulus $(\mathrm{Ep}=$ change in pressure/change in diameter $\times$ diameter in a reference state) $[56,69]$. Whereas this parameter is clearly more reliable and valid than the distensibility mentioned above, more advanced mechanical parameters as outlined previously should be considered [10]. Although these biomechanical metrics are 

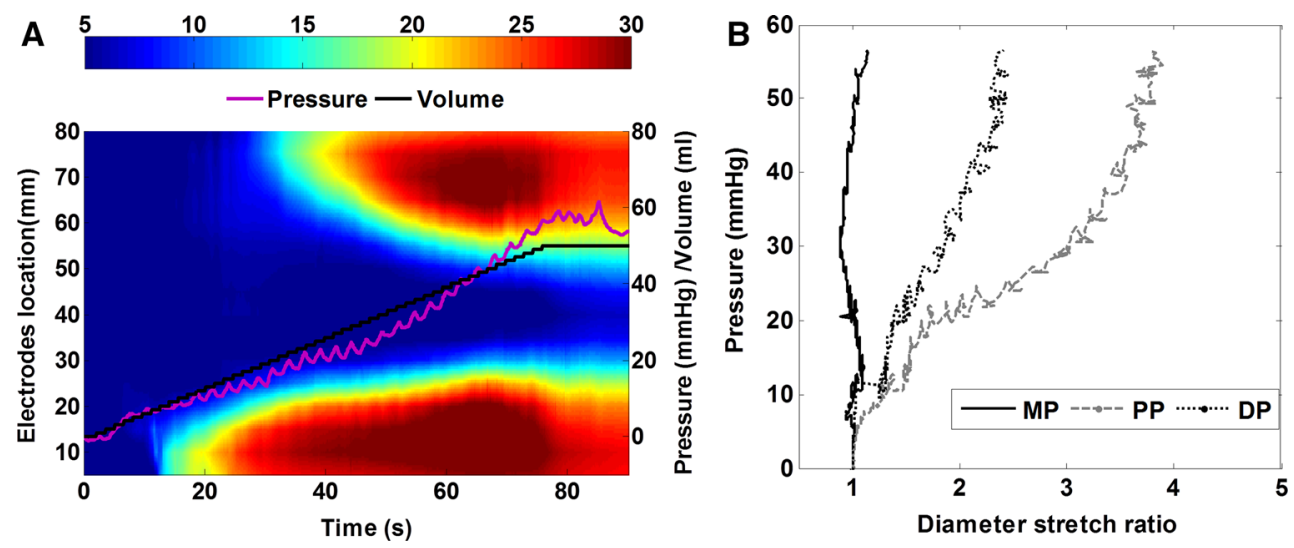

Fig. 7 Left: Spatiotemporal diameter map of functional lumen imaging probe (FLIP) measurement in the anal canal during inflation. The diameter was estimated from the recorded cross-sectional areas. Colors changing from blue to red illustrate diameter increase (maximum diameter $30 \mathrm{~mm}$ ). Each column depicts the configuration of the anal canal at a specific time and each row depicts the change in diameter over time at a particular point along the FLIP. The vertical axis on the left represents the length along the probe and the vertical

axis on the right represents the pressure (purple line) and volume (black line) changes over time. Right: The diameter stretch ratio (diameter/diameter before inflation) and pressure relationship of the middle part $(M P)$, proximal part $(P P)$, and distal part $(D P)$ of the anal canal during inflation up to $50 \mathrm{ml}$ (generated from the data in the left panel). The curve from the middle part is located to the left of the curves from the proximal and distal parts of the anal canal, indicating the middle is the stiffest part of the anal canal

important, this is not always obvious to busy clinicians, who are under time pressure to make rapid clinical decisions. However, conclusions about the tissue properties from these simple measures may be erroneous.

It has become popular to describe these pressure/CSA relationships as a distensibility index, where the index is calculated for the narrowest point in the sphincter [33]. Despite the fact that important data obtained from other parts of the sphincter may not be considered, this approach makes sense from a fluid mechanical point of view, since the narrowest cross section is a very important determinant of the resistance to flow.

\section{Conclusion and perspectives}

The various uses of the FLIP reviewed here suggest it may have a role superior to that of traditional measurement techniques, in particular manometry and the Dent sleeve, and may be an improvement on previous impedance planimetry investigations in the GI tract. The greatest advantage of the FLIP is the ability to measure the mechanical response to distension along the whole sphincter. This is clearly an advantage, since the structure and surrounding tissues are heterogeneously distributed along the longitudinal axis. Variations in distensibility along the sphincter can be explained by the heterogeneous anatomy and active muscle function of the sphincters [39, 54, 56, 57, 69]. In addition to assessment of sphincter wall heterogeneity caused by changes in the mechanical properties and muscle tone, FLIP measurement is also capable of showing alterations caused by diseases, such as achalasia [47, 68],

systemic sclerosis [57], and idiopathic fecal incontinence [56]. These diseases are characterized by significant tissue reconstruction and remodeling, including increased collagen synthesis, dysmotility, and altered wall structure, which are all associated with the altered pressure-diameter relation [10].

In the UES, the FLIP has the potential to revolutionize how we diagnose dysfunction. Moreover, although the FLIP will likely never replace the role of fluoroscopy in dysphagia, it has the potential to complement fluoroscopy and the role of high-resolution manometry in elaborating and deepening our understanding and treatment of swallow-related disorders in the UES.

The FLIP has shown great potential as a means of characterizing the EGJ function in patients with GERD and EoE and for predicting the outcome of surgery in these patient groups. It has come to have an established role and strong association with developing natural orifice transendoscopic surgery for treating achalasia, particularly for Heller myotomy and POEM. However, randomized studies with the FLIP before, after, and as follow-up to these established physical treatment techniques for managing EGJ disease will need to be performed before scientific conclusions can be drawn.

Work in the anorectal region with the FLIP suggests that we have a lot to learn about the complex interaction of the muscle groups, which control retention of feces and defecation. However, it is clear that FLIP measurements can contribute to our knowledge of dysfunction in this region. It will be up to a wider range of pioneering investigators to develop more protocols and build on the work mentioned here to accept or reject a future role for the FLIP in the 
evaluation of the anorectal region and how the FLIP can complement existing motility and imaging assessment techniques.

The anatomy and functioning of GI sphincters is fairly complex. The beauty of the complexity lies in the fact that it provides a myriad of possibilities for novel surgical, endoscopic, and pharmacologic approaches to treat sphincter dysfunction [31]. FLIP measurement provides a comprehensive evaluation of sphincter resistance to mechanical distension. However, to reveal the individual mechanical contributions from each part and component of the sphincter, studies introducing a real constitutive equation with mechanical parameters from different muscle layers and collagen fibers should be considered in future FLIP-based analysis of GI sphincters [70, 71].

All of the authors of this review have in some way shaped the introduction of this technique into clinical practice, and most have had an initial positive experience. This may deem the work biased or narrow in some way. Nevertheless, as a distension technique, it generates enough interest that we challenge the community of active GI functional and motility researchers to develop their own experience with the technique and to document it. For many years, with few exceptions, tests of GI function have involved measurement principles that try to measure function without interfering with it. As a rule, the FLIP is the only measurement tool that directly tries to measure and understand function in valvular or narrowing luminal regions by challenging them through distension. Whether, as a technique, it contributes to our long-term management of any of the conditions mentioned in this review remains to be seen. However, we have no doubt that the knowledge gained in these and other studies will contribute in some way toward a better understanding of function in the GI tract.

Acknowledgments The authors thank Matias Nilsson for the creation of Fig. 1. Donghua Liao was partially supported by the Karen Elise Jensen Foundation (project no. 903959). Hans Gregersen was partially supported by grants from the Chongqing Science and Technology Commission (cstc2013kjrc-ljrccj10003) and the National "111 Plan" Base (B06023).

Conflict of interest The authors declare that they have no conflict of interest.

\section{References}

1. Boron WF, Boulpaep EL. Gastrointestinal motility. Philadelphia: Saunders; 2009.

2. Liu J, Parashar VK, Mittal RK. Asymmetry of lower esophageal sphincter pressure: is it related to the muscle thickness or its shape? Am J Physiol. 1997;272(6 Pt 1):G1509-17.

3. Dent J. A new technique for continuous sphincter pressure measurement. Gastroenterology. 1976;71(2):263-7.
4. Nicodème F, Lin Z, Pandolfino JE, et al. Esophagogastric junction pressure morphology: comparison between a station pullthrough and real-time 3D-HRM representation. Neurogastroenterol Motil. 2013;25(9):e591-8.

5. Gotley DC, Barham CP, Miller R, et al. The sphinctometer: a new device for measurement of lower oesophageal sphincter function. Br J Surg. 1991;78(8):933-5.

6. McMahon BP, Drewes AM, Gregersen H. Functional oesophagogastric junction imaging. World $\mathrm{J}$ Gastroenterol. 2006;12(18):2818-24.

7. Gregersen H, Stodkilde-Jorgensen H, Djurhuus JC, et al. The four-electrode impedance technique: a method for investigation of compliance in luminal organs. Clin Phys Physiol Meas. 1988;9(Suppl A):61-4.

8. Gregersen H, Sørensen S, Sørensen SM, et al. Measurement of anal cross-sectional area and pressure during anal distension in healthy volunteers. Digestion. 1991;48(2):61-9.

9. Patel RS, Rao SS. Biomechanical and sensory parameters of the human esophagus at four levels. Am J Physiol. 1998;275(2 Pt 1):G187-91.

10. Gregersen H. Biomechanics of the gastrointestinal Tract. London: Springer; 2002.

11. Shaker R, Bardan E, Gu C, et al. Effect of lower esophageal sphincter tone and crural diaphragm contraction on distensibility of the gastroesophageal junction in humans. Am J Physiol Gastrointest Liver Physiol. 2004;287(4):G815-21.

12. Pandolfino JE, Shi G, Curry J, et al. Esophagogastric junction distensibility: a factor contributing to sphincter incompetence. Am J Physiol Gastrointest Liver Physiol. 2002;282(6):G1052-8.

13. Liu J, Takeda T, Dogan I, et al. Oesophago-gastric junction opening function: assessment using ultrasound imaging and the effects of atropine. Neurogastroenterol Motil. 2006;18(5):376-84.

14. Andersen IS, Gregersen H, Buntzen S, et al. New probe for the measurement of dynamic changes in the rectum. Neurogastroenterol Motil. 2004;16(1):99-105.

15. McMahon B, Frøkjaer J, Drewes A, et al. A new measurement of oesophago-gastric junction competence. Neurogastroenterol Motil. 2004;16(5):1-4.

16. McMahon B, Frøkjaer J, Liao D, et al. A new technique for evaluating sphincter function in visceral organs: application of the functional lumen imaging probe (FLIP) for the evaluation of the oesophago-gastric junction. Physiol Meas. 2005;26(5):823-36.

17. McMahon BP, Frøkjaer JB, Kunwald P, et al. The functional lumen imaging probe (FLIP) for evaluation of the esophagogastric junction. Am J Physiol Gastrointest Liver Physiol. 2007;292(1):G377-84.

18. Gregersen H, Kraglund K, Djurhuus JC. Variations in duodenal cross-sectional area during the interdigestive migrating motility complex. Am J Physiol. 1990;259(1 Pt 1):G26-31.

19. Gregersen H, Gravesen F, Olesen SS, Drewes AM, McMahon B. New high-resolution functional luminal imaging system for the oesophago-gastric junction. Abstract and poster. UEGW. 2009.

20. Stoeckli SJ, Huisman TAGM, Seifert B, et al. Interrater reliability of videofluoroscopic swallow evaluation. Dysphagia. 2003;18(1):53-7.

21. Rugiu MG. Role of videofluoroscopy in evaluation of neurologic dysphagia. Acta Otorhinolaryngol Ital. 2007;27(6):306-16.

22. Miller L, Clavé P, Farré R, et al. Physiology of the upper segment, body, and lower segment of the esophagus. Ann N Y Acad Sci. 2013;1300:261-77.

23. Karaho T, Satoh T, Nakajima J, et al. Can mano-videoendoscopy substitute for videofluorography in evaluation of upper esophageal sphincter function? Acta Otolaryngol. 2015;135(2):187-92.

24. McMahon B-P. Do we really understand the role of the oesophagogastric junction in disease? World $\mathrm{J}$ Gastroenterol. 2009;15(2):144. 
25. Regan J, Walshe M, Rommel N, et al. A new evaluation of the upper esophageal sphincter using the functional lumen imaging probe: a preliminary report. Dis Esophagus. 2013;26(2):117-23.

26. Regan J, Walshe M, Rommel N, et al. New measures of upper esophageal sphincter distensibility and opening patterns during swallowing in healthy subjects using EndoFLIP ${ }^{\circledR}$. Neurogastroenterol Motil. 2013;25(1):e25-34.

27. Regan J, Walshe M, Timon C, et al. Endoflip ${ }^{\circledR}$ evaluation of pharyngo-oesophageal segment tone and swallowing in a clinical population: a total laryngectomy case series. Clin Otolaryngol. 2015;40:121-9.

28. Fornari F, Gurski R, Navarini D, et al. Clinical utility of endoscopy and barium swallow X-ray in the diagnosis of sliding hiatal hernia in morbidly obese patients: a study before and after gastric bypass. Obes Surg. 2010;20(6):702-8.

29. Fox M, Sweis R. Future directions in esophageal motility and function-new technology and methodology. Neurogastroenterol Motil. 2012;24(Suppl 1):1-9.

30. Curcic J, Fox M, Kaufman E, et al. Gastroesophageal junction: structure and function as assessed by using MR imaging. Radiology. 2010;257(1):115-24.

31. Mittal RK, Balaban DH. The esophagogastric junction. N Engl J Med. 1997;336(13):924-32.

32. Beaumont H, Gondrie JJ, McMahon BP, et al. Stepwise radiofrequency ablation of Barrett's esophagus preserves esophageal inner diameter, compliance, and motility. Endoscopy. 2009;41(1):2-8.

33. Kwiatek M, Pandolfino JE, Hirano I, et al. Esophagogastric junction distensibility assessed with an endoscopic functional luminal imaging probe (EndoFLIP). Gastrointest Endosc. 2010;72(2):272-8.

34. Kwiatek MA, Kahrilas PJ, Soper NJ, et al. Esophagogastric junction distensibility after fundoplication assessed with a novel functional luminal imaging probe. $\mathrm{J}$ Gastrointest Surg. 2010;14(2):268-76.

35. Ilczyszyn A, Botha AJ. Feasibility of esophagogastric junction distensibility measurement during Nissen fundoplication. Dis Esophagus. 2014;27(7):637-44.

36. Nathanson LK, Brunott N, Cavallucci D. Adult esophagogastric junction distensibility during general anesthesia assessed with an endoscopic functional luminal imaging probe $\left(\right.$ EndoFLIP $\left.^{\circledR}\right)$. Surg Endosc. 2012;26(4):1051-5.

37. Hoppo T, McMahon B, Witteman B, et al. Functional lumen imaging probe to assess geometric changes in the esophagogastric junction following endolumenal fundoplication. J Gastrointest Surg. 2011;15(7):1-9.

38. Rinsma NF, Smeets FG, Bruls DW, et al. Effect of transoral incisionless fundoplication on reflux mechanisms. Surg Endosc. 2014;28(3):941-9.

39. Lin Z, Nicodème F, Boris L, et al. Regional variation in distal esophagus distensibility assessed using the functional luminal imaging probe (FLIP). Neurogastroenterol Motil. 2013;25(11):e765-71.

40. Fukazawa K, Furuta K, Adachi K, et al. Effects of mosapride on esophageal motor activity and esophagogastric junction compliance in healthy volunteers. J Gastroenterol. 2014;49(9):1307-13.

41. Lottrup C, McMahon BP, Ejstrud P, et al. Esophagogastric junction distensibility in hiatus hernia. Dis Esophagus. 2015. doi:10.1111/dote. 12344 .

42. Tucker E, Sweis R, Anggiansah A, et al. Measurement of esophago-gastric junction cross-sectional area and distensibility by an endolumenal functional lumen imaging probe for the diagnosis of gastro-esophageal reflux disease. Neurogastroenterol Motil. 2013;25(11):904-10.
43. Kwiatek M, Hirano I, Kahrilas PJ, et al. Mechanical properties of the esophagus in eosinophilic esophagitis. Gastroenterology. 2011;140(1):82-90.

44. Nicodème F, Hirano I, Chen J, et al. Esophageal distensibility as a measure of disease severity in patients with eosinophilic esophagitis. Clin Gastroenterol Hepatol. 2013;11(9):1101-7.e1.

45. Rieder E, Swanström L, Perretta S, et al. Intraoperative assessment of esophagogastric junction distensibility during per oral endoscopic myotomy (POEM) for esophageal motility disorders. Surg Endosc. 2013;27(2):400-5.

46. Rohof W, Hirsch D, Kessing BF, et al. Efficacy of treatment for patients with achalasia depends on the distensibility of the esophagogastric junction. Gastroenterology. 2012;143(2):1-8.

47. Pandolfino JE, de Ruigh A, Nicodème F, et al. Distensibility of the esophagogastric junction assessed with the functional lumen imaging probe $\left(\right.$ FLIP $\left.^{\mathrm{TM}}\right)$ in achalasia patients. Neurogastroenterol Motil. 2013;25(6):496-501.

48. Teitelbaum E, Boris L, Arafat F, et al. Comparison of esophagogastric junction distensibility changes during POEM and Heller myotomy using intraoperative FLIP. Surg Endosc. 2013;27(12):4547-55.

49. Teitelbaum EN, Soper NJ, Pandolfino JE, et al. Esophagogastric junction distensibility measurements during Heller myotomy and POEM for achalasia predict postoperative symptomatic outcomes. Surg Endosc. 2015;29(3):522-528.

50. Familiari P, Gigante G, Marchese M, et al. EndoFLIP system for the intraoperative evaluation of peroral endoscopic myotomy. United Eur Gastroenterol J. 2014;2(2):77-83.

51. Teitelbaum EN, Soper NJ, Pandolfino JE, et al. An extended proximal esophageal myotomy is necessary to normalize EGJ distensibility during Heller myotomy for achalasia, but not POEM. Surg Endosc. 2014;28(10):2840-7.

52. Perretta S, Dallemagne B, McMahon B, et al. Video. Improving functional esophageal surgery with a "smart" bougie: endoflip. Surg Endosc. 2011;25(9):3109.

53. Harris LD, Winans CS, Pope CE. Determination of yield pressures: a method for measuring anal sphincter competence. Gastroenterology. 1966;50(6):754-60.

54. Luft F, Fynne L, Gregersen H, et al. Functional luminal imaging probe: a new technique for dynamic evaluation of mechanical properties of the anal canal. Tech Coloproctol. 2012;16(6):451-7.

55. Alqudah M, Gregersen H, Drewes A, et al. Evaluation of anal sphincter resistance and distensibility in healthy controls using EndoFLIP (C). Neurogastroenterol Motil. 2012;24(12):e591-9.

56. Sørensen G, Liao D, Lundby L, et al. Distensibility of the anal canal in patients with idiopathic fecal incontinence: a study with the functional lumen imaging probe. Neurogastroenterol Motil. 2014;26(2):255-63.

57. Fynne L, Luft F, Gregersen H, et al. Distensibility of the anal canal in patients with systemic sclerosis: a study with the functional lumen imaging probe. Colorectal Dis. 2013;15(1):e40-7.

58. Thoua NM, Schizas A, Forbes A, et al. Internal anal sphincter atrophy in patients with systemic sclerosis. Rheumatology (Oxf). 2011;50(9):1596-602.

59. Abdool Z, Sultan AH, Thakar R. Ultrasound imaging of the anal sphincter complex: a review. Br J Radiol. 1015;2012(85):865-75.

60. Pandolfino JE, Kahrilas PJ. American Gastroenterological Association medical position statement: clinical use of esophageal manometry. Gastroenterology. 2005;128(1):207-8.

61. Hongo M, Machida T. (New diagnostic approaches for esophageal motility disorders using high-resolution manometry). Nihon Shokakibyo Gakkai Zasshi. 2012;109(5):703-9.

62. Dobben AC, Felt-Bersma RJF, ten Kate FJW, et al. Cross-sectional imaging of the anal sphincter in fecal incontinence. AJR Am J Roentgenol. 2008;190(3):671-82. 
63. Murray JA, Clouse RE, Conklin JL. Components of the standard oesophageal manometry. Neurogastroenterol Motil. 2003;15(6): 591-606.

64. Sajid MS, Khatri K, Siddiqui MRS, et al. Endo-anal ultrasound versus endo-anal magnetic resonance imaging for the depiction of external anal sphincter pathology in patients with faecal incontinence: a systematic review. Magy Seb. 2010;63(1):9-15.

65. Gregersen H, Liao D, Pedersen J, et al. A new method for evaluation of intestinal muscle contraction properties: studies in normal subjects and in patients with systemic sclerosis. Neurogastroenterol Motil. 2007;19(1):11-9.

66. Kim YS, Weinstein M, Raizada V, et al. Anatomical disruption and length-tension dysfunction of anal sphincter complex muscles in women with fecal incontinence. Dis Colon Rectum. 2013;56(11):1282-9.
67. Zannoli R, Corazza I, Di Donna M, et al. The biomechanical behavior of sphincters: how the theory and the practice mismatch. J Mech Med Biol. 2005;05(03):477-84.

68. McMahon B, Frøkjaer J, Kunwald P, et al. The functional lumen imaging probe (FLIP) for evaluation of the esophagogastric junction. Am J Physiol Gastrointest Liver Physiol. 2007;292(1):1-9.

69. Hee L, Liao D, Sandager P, et al. Cervical stiffness evaluated in vivo by endoflip in pregnant women. PLoS One. 2014;9(3):e91121.

70. Liao D, Hee L, Sandager P, et al. Identification of biomechanical properties in vivo in human uterine cervix. J Mech Behav Biomed Mater. 2014;39:27-37.

71. Boron WF, Boulpaep EL. Biomechanics of soft tissue in cardiovascular systems. Vienna: Springer; 2003. 